\title{
Mapeo del daño en bosques incendiados de Chile central, mediante el modelado de índices espectrales ex-ante y ex-post
}

\author{
Mapping damage on forests burnt in Central Chile \\ by modelling ex-ante and ex-post spectral indices
}

\author{
Marco A Peña ${ }^{\text {a,b* }}$, Gonzalo Martínez ${ }^{\text {a }}$ \\ *Autor de correspondencia: ${ }^{a}$ Universidad Alberto Hurtado, Departamento de Geografía, \\ Santiago, Chile, tel.: 56-2-8897782,mapena@uahurtado.cl \\ ${ }^{\mathrm{b}}$ Friedrich Schiller University, Department of Geography, Jena, Germany.
}

\begin{abstract}
SUMMARY
This study estimated the severity of Nilahue-Barahona and Las Máquinas wildfires, occurred in central Chile during the summer of 2016-17, by an empirical-statistical modelling based on pre- and post-fire arithmetic differences of spectral indices sensitive to vigor, turgor and calcination states of vegetation. By doing this, map of damages were created to aid the efficient management and ecological restoration of disturbed forestry ecosystems. The index differences were calculated from Sentinel-2 satellite images, acquired anually in the summers spanning from 2016 to 2019. The resulting nine index-derived differences were used as predictors of burn severity, field-measured during the summer of 2019 using the CBI (composite burn index) method, into a linear stepwise regression that allowed for selecting those with the highest predictability. CBI yielded low correlations as its calculation includes low vegetation strata largely recovered at the time of the field data collection. However, when overstory field data were used alone, correlations increased ( $70 \%$ of the data $\geq 0.80, P<0.05$ ). This was because this stratum was still appreciably damaged during the field campaign, along with its best representation from the image planimetry. The burn severity of both wildfires was mapped using the overstory data as regressand in a model based on NDWI ex-ante-2019$, \mathrm{NDWI}_{\text {ex-ante-2018}}, \mathrm{NBR}_{\text {ex-ante-2018}}$ and $\mathrm{NBR}_{\text {ex-ante-2017 }}$ differences $\left(R^{2} \mathrm{ad}=0.77, \mathrm{RMSE}=0.35\right)$.
\end{abstract}

Key words: wildfires, Sentinel-2, vegetation spectral indices, stepwise regression, composite burn index.

\section{RESUMEN}

Este trabajo estimó la severidad de los incendios forestales Nilahue-Barahona y Las Máquinas, ocurridos en Chile central en el verano de 2016-17, a partir del modelado empírico-estadístico de diferencias aritméticas entre fechas pre- y post-incendio de índices espectrales sensibles a los estados de vigor, turgencia y calcinación vegetal. A partir de lo anterior, fueron creadas cartografías de daño que puedan orientar con mayor eficiencia el manejo y restauración ecológica de los ecosistemas forestales perturbados. Las diferencias de índices fueron calculadas desde imágenes satelitales Sentinel-2, adquiridas anualmente en los veranos de 2016 a 2019 e ingresadas como predictoras de la severidad del incendio; medida en terreno en el verano de 2019 mediante el método CBI (composite burn index), en una regresión lineal paso a paso que permitió seleccionar aquellas con mayor predictibilidad. El CBI arrojó bajas correlaciones, debido a que su cálculo incluye estratos vegetales inferiores, en su mayoría recuperados al momento de colección de los datos de terreno. Sin embargo, cuando solo fueron empleados los datos medidos en el dosel arbóreo superior, las correlaciones aumentaron ( $70 \%$ de los datos $\geq 0,80 ; P<0,05)$. Esto se debió a que este estrato aún permanecía apreciablemente dañado durante la campaña de terreno, junto a su mejor representación desde la planimetría de las imágenes. La severidad de ambos incendios tomando los datos del dosel arbóreo superior como regresando, fue mapeada utilizando un modelo basado en las diferencias NDWI $\mathrm{NDWI}_{\text {ex-ante-2018 }}, \mathrm{NBR}_{\text {ex-ante-2018 }} \mathrm{y} \mathrm{NBR}_{\text {ex-ante-2017 }}\left(R^{2}\right.$ ajustado $\left.=0,77 ; \mathrm{RMSE}=0,35\right)$.

Palabras clave: incendios forestales, Sentinel-2, índices espectrales de vegetación, regresión paso a paso, índice compuesto de quemas.

\section{INTRODUCCIÓN}

Es ampliamente reconocido que los incendios pueden producir severos disturbios en los ecosistemas forestales. Entre estos: destrucción de biodiversidad, fragmentación de hábitat, erosión y esterilización de suelos, polución del agua y la atmósfera, y liberación de dióxido de carbono
(Chuvieco 1999). Aunque la mayoría de los incendios forestales son causados por acción humana, la tendencia global de incremento en las temperaturas y sequías, influenciada por el cambio climático, contribuye a aumentar su frecuencia e intensidad (Westerling et al. 2006, González et al. 2011). Esto ocurre especialmente en regiones sujetas a veranos cálidos y secos prolongados, como las 
insertas en climas de tipo Mediterráneo, donde la triada de fuego (combustible, clima y topografía) se potencia (DíazDelgado 2000, González et al. 2011, Díaz-Hormazábal y González 2016, Plana et al. 2016).

Evaluar las secuelas ecológicas posteriores a un incendio forestal (y que definen su severidad), así como monitorear la progresión de recuperación de los bosques afectados, son tareas clave para orientar acciones sitioespecíficas de corto y largo plazo de manejo y restauración ecológica de un ecosistema forestal incendiado (Key y Benson 2006, Lentile et al. 2006). En este sentido, la teledetección óptica satelital para la observación terrestre destaca como una herramienta costo-eficiente para capturar y analizar datos relacionados con el daño causado por un incendio forestal, ofreciendo una representación espacialmente sinóptica y periódica de vastas áreas afectadas, en la forma de imágenes capturadas en distintas porciones del espectro electromagnético reflejado (i.e., bandas espectrales) (Lentile et al. 2006, Gitas et al. 2012).

Una aproximación metodológica ampliamente documentada en la literatura científica, corresponde al cálculo de índices espectrales; que resultan de combinar aritméticamente bandas sensibles al estado de la vegetación, desde imágenes adquiridas en fechas pre- y post-incendio, que al ser restadas proveen una medida relativa de severidad de la perturbación, en adelante, diferencias de índices (Chuvieco et al. 2002, Key y Benson 2006, Escuín et al. 2008, Sever et al. 2012, Ryu et al. 2018). Así por ejemplo, Chen et al. (2011) elaboran un mapa de recuperación de un bosque incendiado de Pino ponderosa (Pinus ponderosa Douglas ex C.Lawson), mediante una regresión múltiple en árbol $\left(R^{2}=0,85\right)$, que incorpora varios tipos de diferencias de índices como predictores de la severidad del incendio, a su vez, medida en terreno mediante el índice compuesto de quemas (composite burn index, CBI); un método observacional de uso frecuente para relacionar el daño biofísico producido por un incendio con diferencias de índices espectrales sensibles a la calcinación vegetal (Key y Benson 2006, French et al. 2008, De Santis y Chuvieco 2009). De forma similar, Veraverbeke et al. (2011) y Arellano et al. (2017) modelan la severidad de incendios ocurridos en bosques mediterráneos mediante regresiones, tanto lineales como polinomiales, entre el CBI y una diferencia de índice sensible a la vegetación quemada $\left(R^{2}=0,69\right.$ y 0,65 , respectivamente).

La habilidad de un ecosistema forestal incendiado para regenerarse y alcanzar su condición primaria, es decir, su capacidad de resiliencia, depende de la severidad con que su biomasa fue calcinada, y de la subsecuente respuesta de variables como: reclutamiento de semillas, capacidad de rebrote, colonización de especies nativas y exóticas, entre otras (Lentile et al. 2006, Keeley 2009, Chen et al. 2011). En este contexto, cabe destacar que los datos provistos por las diferencias de índices son más bien sensibles a cambios en el monto total de biomasa calcinada o recuperada, y no necesariamente al restablecimiento de otros atributos ecológicos del ecosistema forestal perturbado, como aquellos relacionados con su composición y estructura (Bastos et al. 2011, Peña y Ulloa 2017). Por otro lado, aunque la relación de las diferencias de índices con mediciones asociadas a la severidad de un incendio como el CBI, ha sido ampliamente abordada en la literatura científica, esta no siempre es consistente, existiendo varios estudios que reportan débiles correlaciones entre ambas fuentes de datos, a causa de singularidades en las condiciones ecológicas de las áreas afectadas, así como en la naturaleza y comportamiento del fuego mismo (Zhu et al. 2006, French et al. 2008). Debido a lo anterior, la correcta interpretación de los valores adimensionales arrojados por las diferencias de índices en términos de la respuesta ecológica de un ecosistema forestal a un incendio dado, debe establecerse empíricamente (Key y Benson 2006, Lentile et al. 2006, De Santis y Chuvieco 2009).

En Chile, desde diciembre de 2016 a febrero de 2017, centenares de incendios se extendieron entre las regiones de Coquimbo y Los Lagos $\left(32-38^{\circ} \mathrm{S}, 70-73^{\circ} \mathrm{W}\right)$, propiciados por características climáticas inusualmente favorables para la propagación e intensidad del fuego ("factor 30-3030 ", i.e., temperatura sobre $30^{\circ} \mathrm{C}$, vientos de hasta $30 \mathrm{~km} / \mathrm{h}$ y humedad inferior a $30 \%$ ) (CONAF 2017, Pérez Mato 2017). El descontrol de estos derivó en un mega incendio o "tormenta de fuego", que por su inusitada magnitud dio origen a una nueva categoría de clasificación; el primero en el mundo de la llamada "sexta generación". Durante el evento, se produjeron dos de los incendios forestales más grandes de la historia de Chile, conocidos como Nilahue-Barahona (ubicado entre las latitudes $34^{\circ} 05^{\prime}$ y $34^{\circ} 75^{\prime} \mathrm{S}$ ), en la región del Libertador General Bernardo O'Higgins, y Las Máquinas (ubicado entre las latitudes $35^{\circ} 05^{\prime}$ y $35^{\circ} 51^{\prime} \mathrm{S}$ ), en la región del Maule (CONAF 2017). Si bien estudios previos emplean índices espectrales y sus diferencias como indicadores de la severidad de ambos incendios (CONAF 2017, Bowman et al. 2018, Peña y Martínez 2019), ninguno las modela empíricamente sobre la base de mediciones que le otorguen una interpretabilidad en términos de daño biofísico. Tal tarea demanda la adecuada colección de datos de terreno, para relacionarlos con el tipo de índice espectral más sensible a la severidad del incendio. Sin embargo, la selección a priori de un predictor puede condicionar el bajo rendimiento del modelo, en especial, si el incendio ocurrió hace algunos años atrás y los datos de terreno son medidos en la actualidad, reflejando más bien la recuperación de los bosques afectados. Ante esto, es preferible diseñar un modelo que a partir de un conjunto inicial de predictores representativos de la trayectoria postincendio de un área dada, seleccione solo aquellos que sumen la mayor predictibilidad de la variable de interés, tal como es demostrado en estudios de caracterizaciones estructurales de bosque nativo (Peña et al. 2012) o clasificación de cultivos agrícolas (Peña et al. 2017).

A partir de lo anterior, este estudio plantea como interrogante la factibilidad de diseñar un modelo empírico-es- 
tadístico de severidad de incendio para áreas forestadas de Chile central afectadas por la "tormenta de fuego", capaz de transferirse a una cartografía que contribuya a orientar y planificar con mayor eficiencia el manejo y restauración ecológica de sus ecosistemas forestales perturbados. La hipótesis esbozada a este respecto, plantea que tal diseño es posible a partir de la selección de un conjunto de diferencias de índices que retraten distintas variables de estado vegetal a lo largo de la trayectoria post-incendio del área de interés. De esta forma, se define como objetivo estimar la severidad de los incendios forestales Nilahue-Barahona y Las Máquinas a partir de diferencias de índices espectrales de estado vegetal. Para esto, se diseña un modelo de regresión paso a paso, que busca predecir la severidad de ambos incendios medida en terreno mediante el CBI, a partir del cálculo de diferencias de índices vinculados a los estados de vigor, turgencia y calcinación de la vegetación, y derivados desde imágenes satelitales Sentinel-2, adquiridas anualmente en veranos comprendidos entre 2016 y 2019.

\section{MÉTODOS}

Área de estudio. Dos incendios forestales ocurridos durante el verano de 2016-17, en Chile central fueron definidos como áreas de estudio: Nilahue Barahona y Las Máquinas, cuyos nombres refieren a las localidades donde el fuego se inició o propagó. La primera, ubicada en la región del Libertador General Bernardo O'Higgins, abarca un área de 488 $\mathrm{km}^{2}$ (figura 1A). La segunda, ubicada en la región del Maule, abarca un área de $1.944 \mathrm{~km}^{2}$ (figura 1B). Ambas presentan una alta presencia de coberturas forestales, distribuidas principalmente a lo largo de la Cordillera de la Costa, a una altitud promedio aproximada de $400 \mathrm{~m}$ s.n.m. y sujetas a un clima predominantemente Mediterráneo (i.e. veranos secos y extensos y precipitaciones concentradas en invierno). En Nilahue-Barahona las plantaciones forestales y el bosque nativo alcanzan el 44,92 y $55,08 \%$ respectivamente, de la totalidad del área forestada, mientras que en Las Máquinas esos porcentajes corresponden a 78,89 y 16,39 (además de $4,72 \%$ de bosque mixto). En ambas áreas las plantaciones forestales corresponden básicamente a monocultivos de Pino Radiata (Pinus radiata D. Don), mientras que el bosque nativo se compone mayoritariamente de formaciones compuestas por Peumo (Cryptocarya alba (Molina) Looser), Quillay (Quillaja saponaria Molina) y Litre (Lithraea caustica (Molina) Hook. et Arn.), y en menor proporción Roble (Nothofagus obliqua (Mirb.) Oerst.) y Hualo (Nothofagus glauca (Phil.) Krasser 1896) (IDE 2018).

Materiales. Un conjunto de cuatro imágenes Sentinel-2 de cada área de estudio, adquiridas durante veranos de años pre- y post-incendio (cuadro 1), fue descargado desde el Centro de Acceso Abierto de Copernicus, perteneciente a la Agencia Espacial Europea (ESA 2020).

Cuadro 1. Fechas de adquisición de las imágenes Sentinel-2 usadas en cada área de estudio.

Acquisition dates of Sentinel-2 images used on each study area.

\begin{tabular}{llr}
\hline \multirow{2}{*}{ Área de estudio } & \multicolumn{2}{c}{ Fecha de adquisición de la imagen } \\
\cline { 2 - 3 } & Pre-incendio & Post-incendio \\
\hline
\end{tabular}

Nilahue-Barahona Diciembre 20, 2016 Febrero 18, 2018

Febrero 23, 2019

Marzo 20, 2017

Las Máquinas $\quad$ Enero 19, $2017 \quad$ Marzo 15, 2018

Marzo 20, 2019
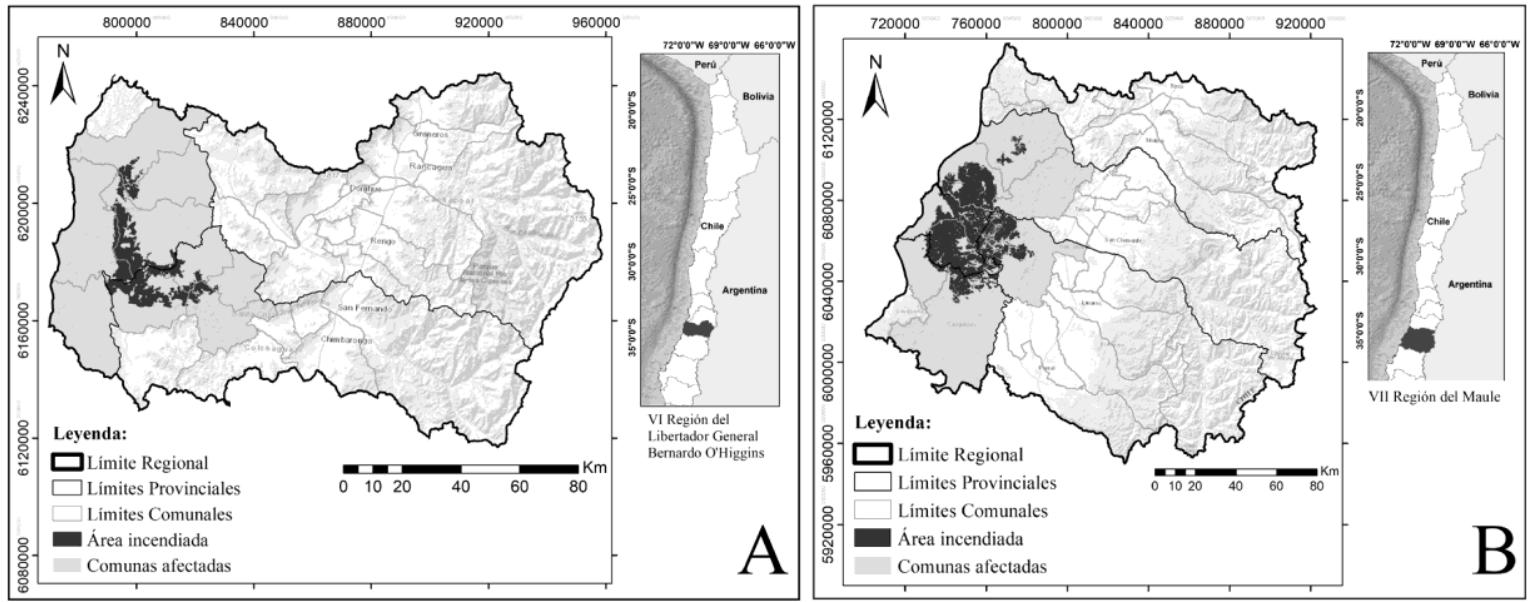

Figura 1. Contexto político-administrativo de las áreas de estudio Nilahue-Barahona (A) y Las Máquinas (B).

Political-administrative context of Nilahue-Barahona (A) and Las Máquinas (B) study areas. 
Las imágenes Sentinel-2 son adquiridas por un sensor óptico de empuje (Multispectral Instrument, MSI), que abarca 13 bandas a lo largo de las longitudes de onda visible e infrarrojas del espectro electromagnético (cuadro 2). El producto descargado (L2A) contiene reflectancias superficiales ortorectificadas en el sistema de proyección UTM (Universal Transversal de Mercator), datum WGS84 (World Geodetic System 1984), de modo que para los propósitos de este estudio no requirió de procedimientos adicionales de corrección geométrica y radiométrica. Para su posterior procesamiento, las bandas de cada imagen fueron espacialmente remuestreadas al tamaño de píxel más pequeño de Sentinel-2 $(10 \mathrm{~m})$ mediante el método de vecino más cercano.

Métodos. Tres tipos de índices espectrales, cada uno sensible a una variable de estado vegetal específica, fueron obtenidos desde cada una de las cuatro imágenes (cuadro 3) mediante el procesador de imágenes ENVI ${ }^{\circledR}$ (ENviron-

Cuadro 2. Principales características técnicas de las imágenes Sentinel-2.

Main technical characteristics of Sentinel-2 images.

\begin{tabular}{cccc}
\hline $\begin{array}{c}\text { Resolución } \\
\text { espacial }(\mathrm{m})\end{array}$ & \# banda & $\begin{array}{c}\text { Longitud de } \\
\text { onda central } \\
(\mathrm{nm})\end{array}$ & $\begin{array}{c}\text { Ancho de } \\
\text { banda } \\
(\mathrm{nm})\end{array}$ \\
\hline \multirow{3}{*}{10} & 2 & 490 & 65 \\
& 3 & 560 & 35 \\
& 4 & 665 & 30 \\
& 8 & 842 & 115 \\
& 5 & 705 & 15 \\
20 & 6 & 740 & 15 \\
& 7 & 783 & 20 \\
& $8 \mathrm{a}$ & 865 & 20 \\
& 11 & 1.610 & 90 \\
& 12 & 2.190 & 180 \\
& 1 & 443 & 20 \\
& 9 & 945 & 20 \\
& 10 & 1.380 & 30 \\
\hline
\end{tabular}

ment for Visualizing Images) v.5.3 (Exelis Visual Information Solutions, Inc., Boulder, EE.UU.). El índice de vegetación de diferencia normalizada (normalized difference vegetation index, NDVI) se construye con las bandas del rojo; sensible a la absorción de radiación que produce la clorofila foliar, e infrarrojo cercano; sensible a la reflexión de radiación que produce la abundancia de follaje sano, por lo que sus valores dan cuenta del estado de vigor o verdor del vegetal (Rouse et al. 1973, Chuvieco et al. 2002). El índice de agua de diferencia normalizada (normalized difference water index, NDWI) y el índice de calcinación normalizado (normalized burn ratio, NBR) emplean la misma banda del infrarrojo cercano en su construcción, además de una banda del infrarrojo de onda corta, que difiere en su posición espectral de acuerdo a la aplicación para la cual cada índice fue originalmente diseñado. En el primer caso, su posición marca la absorción de radiación producida por el agua foliar, por lo que sus valores dan cuenta del estado de turgencia vegetal, mientras que, en el segundo caso, su posición se mueve a longitudes de onda mayores; más estrechamente asociadas al estrés hídrico inducido por el fuego y, por lo tanto, al estado de calcinación vegetal (Hunt et al. 1987, Gao 1996, Key y Benson 2006, Veraverbeke et al. 2011).

Cada índice espectral post-incendio fue restado a su equivalente pre-incendio para obtener una diferencia relativa a la severidad del incendio en ese período. Una campaña de terreno realizada en el verano de 2019, permitió medir dicha variable sobre 35 parcelas de las coberturas forestales de interés, cada una con una condición homogénea dentro de un radio de $20 \mathrm{~m}$ (dos veces el tamaño de píxel de imagen empleado). Estas fueron discrecionalmente ubicadas a través de las plantaciones forestales $(n=24)$ y el bosque nativo $(n=11)$ de las áreas de estudio Nilahue-Barahona $(n$ $=20)$ y Las Máquinas $(n=15)$. Las mediciones fueron hechas mediante el CBI, un índice que resulta de promediar la magnitud de calcinación causada por un incendio en una parcela forestal dada, a partir de la estimación visual de un conjunto de parámetros de daño biofísico del sotobosque (incluyendo el substrato) y los doseles arbóreos superiores, usando una escala de puntuación continua que va desde 0 (sin rasgos de daño por calcinación) a 3 (completamente dañado por calcinación) y que puede discretizarse en tres rangos de daño: $<1$ : bajo; 1-2: moderado y $>2$ : alto (para más detalles metodológicos ver Key y Benson 2006).

Cuadro 3. Índices espectrales de vegetación usados en este estudio. Su formulación refiere al número de banda (b) de Sentinel-2.

Vegetation spectral indices used in this study. Their formulation refers to the Sentinel-2 band (b) number.

\begin{tabular}{lccl}
\hline \multicolumn{1}{c}{ Índice } & Notación & \multicolumn{1}{c}{ Fórmula } & \multicolumn{1}{c}{ Referencia } \\
\hline Índice de vegetación de diferencia normalizada & NDVI & $(\mathrm{b} 8-\mathrm{b} 4) /(\mathrm{b} 8+\mathrm{b} 4)$ & Rouse et al. $(1973)$ \\
Índice de agua de diferencia normalizada & NDWI & $(\mathrm{b} 8-\mathrm{b} 11) /(\mathrm{b} 8+\mathrm{b} 11)$ & Hunt et al. (1987) \\
Índice de calcinación normalizado & NBR & $(\mathrm{b} 8-\mathrm{b} 12) /(\mathrm{b} 8+\mathrm{b} 12)$ & Key y Benson (2006) \\
\hline
\end{tabular}


Las nueve diferencias de índices resultantes fueron correlacionadas mediante el método de Pearson, con tres conjuntos de datos de terreno: (1) las mediciones hechas en el sotobosque y (2) el dosel arbóreo superior por separado, y (3) el CBI que integra a ambas. Una regresión lineal paso a paso (i.e. combinando selección hacia adelante y eliminación hacia atrás de variables) fue realizada empleando como variable regresada el conjunto de datos de terreno que mostró las correlaciones más altas (2) y como regresores las diferencias de índices. En el contexto de incendios forestales, el uso de este tipo de regresión destaca en el modelado de vulnerabilidad de incendios (Oliveira et al. 2018) y de calcinación de copas arbóreas (Kafka et al. 2001). Su formulación es idéntica a la de una regresión lineal múltiple, excepto que incorpora solo aquellos regresores que producen la mayor predicción de la variable regresada (i.e., mejor ajuste de la función lineal). Para esto, los regresores son sumados uno a uno al modelo, seleccionando solo aquellos que producen el mayor incremento adicional del coeficiente de determinación $\left(R^{2}\right)$ ajustado (selección hacia adelante). Una vez sumado el regresor que cumple con tal condición, cualquier otro que no influya en mantener ese nuevo ajuste es removido del modelo (eliminación hacia atrás) (James et al. 2013).

El rendimiento del modelo fue evaluado mediante la raíz del error cuadrático medio (root mean square error, RMSE) obtenida entre los datos de CBI reales (medidos en terreno) y predichos (modelados), a partir de la aplicación de una validación cruzada; particularmente útil cuando el número de datos de la variable regresada $(n)$ es reducido, limitando separar un grupo para propósitos exclusivos de validación del modelo. Este procedimiento particiona $n$ en $k$ grupos, utilizando uno para validar y el resto para entrenar el modelo. Posteriormente, un grupo distinto de datos es empleado en la validación y el resto en el entrenamiento del modelo y así hasta completar $k$ iteraciones, que arrojarán $k$ RMSEs reducibles a un único valor promedio. Sin embargo, dado que la regresión paso a paso busca seleccionar un único modelo a partir de $n$, la separación iterativa de datos de validación podría introducir algunas variaciones en los regresores óptimos que lo conforman, en atención a la estrategia de particionamiento de $n$; especialmente, si este es pequeño en relación con el número total de regresores. Para evitar esto, fue implementada una validación cruzada "dejando uno fuera" (leave-one-out cross-validation, LOOCV), que itera la regresión un número de veces equivalente a $n$, sustrayendo en cada ocasión un dato distinto $(n-1)$, que luego es predicho mediante la regresión de la que fue excluido. La exclusión de un púnico dato en cada iteración tendrá un efecto mínimo en la selección del modelo, a la vez de permitir su validación con datos independientes, para finalmente promediar los $n$ RMSEs obtenidos (James et al. 2013). Estos procedimientos fueron implementados en el software de análisis estadístico R-Project ${ }^{\circledR}$ v.4.0.3 (R Core Team, Viena, Austria), mediante el uso de las librerías MASS (Modern Applied Statistics with $S$ ) y DAAG (Data Analysis and Graphics Data and Functions).

\section{RESULTADOS}

Como cabría esperar, en ambas áreas de estudio las diferencias de índices fueron las más amplias cuando incluyeron la primera fecha post-incendio, reflejándose en sus mayores diferencias promedio (figuras $2 \mathrm{~A}$ y $2 \mathrm{~B}$ ). Tales promedios se estrecharon progresivamente a lo largo de las diferencias de índices que incluyeron las fechas postincendio restantes. En general, las diferencias de índices exhibieron similares patrones temporales, con las plantaciones forestales mostrando promedios más altos en las diferencias de índices que aquellos pertenecientes a bosque nativo o mixto.

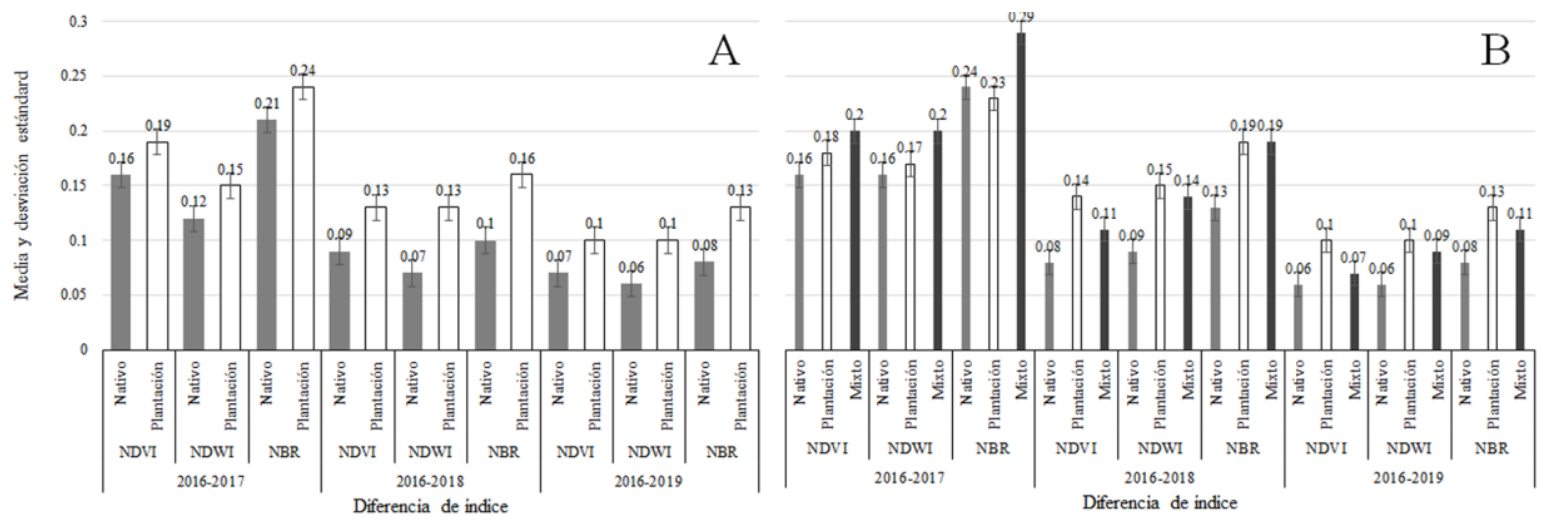

Figura 2. Media y desviación estándar de las diferencias de índices (resta entre la fecha ex-ante y una ex-post) para las coberturas forestales de las áreas Nilahue-Barahona (A) y Las Máquinas (B).

Mean and standard deviation of the index-based differences (subtraction between the ex-ante and ex-post dates) for the forest lands covers of Nilahue-Barahona (A) and Las Máquinas (B) study areas. 
Las diferencias de índices arrojaron coeficientes de correlación notoriamente distintos según los datos de terreno empleados (cuadro 4). En el caso del CBI (i.e., medición promediada de los doseles arbóreos superior e inferior), las correlaciones fueron moderadas ( $r=0,44-0,66$; $P<0,05)$, sin embargo, cuando solo los datos del dosel arbóreo superior fueron considerados, estas incrementaron $(70 \%$ de los datos $\geq 0,80 ; P<0,05)$. En el caso del sotobosque, en cambio, las correlaciones fueron bajas $(\leq 0,35 ; P=\mathrm{ns})$. Similares comportamientos fueron observados cuando los datos fueron divididos de acuerdo al tipo de bosque (datos no mostrados). Se observó que las diferencias de NDWI y el NBR fueron las que arrojaron las mayores correlaciones con los datos del dosel arbóreo superior, especialmente si incluyeron la segunda y tercera fecha post-incendio $(r=0,80-0,86 ; P<0,05)$.

Cuadro 4. Coeficientes de correlación ( $r$ ) para el CBI y los estratos vegetales que componen su medición, según diferencia de índice.

Correlation coefficients $(r)$ for $\mathrm{CBI}$ and the vegetation strata composing its measurement, according to index-derived difference.

\begin{tabular}{clccc}
\hline \multirow{2}{*}{$\begin{array}{c}\text { Resta de } \\
\text { imágenes }\end{array}$} & Estrato vegetal & \multicolumn{3}{c}{$r$} \\
\cline { 3 - 5 } & & NDVI & NDWI & NBR \\
\hline \multirow{2}{*}{ ex-ante-2017 } & Dosel superior & $0,63^{* * *}$ & $0,52^{* *}$ & $0,51^{* *}$ \\
& Sotobosque & $0,27^{\text {ns }}$ & $0,24^{\text {ns }}$ & $0,35^{*}$ \\
& CBI & $0,50^{* * *}$ & $0,44^{* *}$ & $0,48^{* *}$ \\
& Dosel superior & $0,81^{* * *}$ & $0,80^{* * *}$ & $0,80^{* * *}$ \\
ex-ante-2018 & Sotobosque & $0,14^{\mathrm{ns}}$ & $0,10^{\mathrm{ns}}$ & $0,17^{\mathrm{ns}}$ \\
& CBI & $0,54^{* * *}$ & $0,54^{* * *}$ & $0,56^{* * *}$ \\
& Dosel superior & $0,86^{*}$ & $0,86^{* * *}$ & $0,85^{* *}$ \\
ex-ante-2019 & Sotobosque & $0,26^{\mathrm{ns}}$ & $0,15^{\mathrm{ns}}$ & $0,20^{\mathrm{ns}}$ \\
& CBI & $0,66^{*}$ & $0,61^{* * *}$ & $0,64^{* * *}$ \\
\hline
\end{tabular}

$*=P<0,05 ; * *=P<0,01 ; * * *=P<0,001 ; \mathrm{ns}=$ no significativo.

$*=P<0.05, * *=P<0.01, * * *=P<0.001, \mathrm{~ns}=$ non-significant.
A partir de estos resultados, la regresión paso a paso fue empleada con los datos del dosel arbóreo superior de ambas áreas de estudio, arrojando que el modelo con mejor ajuste se compone de los predictores NDWI $\mathrm{NDWI}_{\text {ex-ante-2018}}, \mathrm{NBR}_{\text {ex-ante-2018 }} \mathrm{y} \mathrm{NBR}_{\text {ex-ante-2017 }}\left(R^{2}\right.$ ajustado $=$ $0,77$; RMSE $=0,35)$. A partir de este resultado, un mapa de CBI fue creado para cada área de estudio, cuyos valores fueron agrupados en los tres rangos de daño sugeridos por Key y Benson (2006), autores de este método de medición, a fin de facilitar su interpretación espacial (figuras 3B y 3D). Junto a cada mapa, se añadió su respectiva cartografía de tipos de bosque (figuras 3A y 3C; IDE, 2018), con el propósito de facilitar su relación espacial.

La distribución de valores de CBI predicho fue consistente entre ambas áreas de estudios (cuadro 5), con una alta concentración en torno al rango Moderado tanto en bosque nativo como plantaciones, y una distribución más ponderada entre los rangos Alto y Bajo tanto en bosque nativo como plantaciones.

\section{DISCUSIÓN}

Las diferencias de índices muestran que los bosques de ambas áreas de estudio experimentaron una progresiva recuperación de biomasa tras dos años de ocurridos los incendios. Sin embargo, sin importar el índice espectral, las plantaciones forestales exhiben más bajas recuperaciones en comparación con el bosque nativo, concordando con lo reportado por Bowman et al. (2018) para el conjunto de incendios que afectó a Chile central entre diciembre de 2016 y febrero de 2017, a partir del cálculo de diferencias de índices compuestos de verdor vegetal derivados de imágenes MODIS (Moderate-Resolution Imaging Spectroradiometer). Esto se debe a la alta intensidad de fuego que pueden propiciar las plantaciones en estudio, como resultado de la alta carga de combustible de sus especies dominantes (Pinus radiata y Eucalyptus spp.), siendo este un factor determinante en la inusitada voracidad que alcanzaron estos incendios y su consecuente calcinación de biomasa (Peña-Fernández y Valenzuela-Palma 2004, Bowman et

Cuadro 5. Superficie ocupada por cada rango de CBI según tipo de bosque en las áreas de estudio.

Surface occupied by each CBI range according to forest type on the study areas.

\begin{tabular}{lcccccccccc}
\hline & \multicolumn{9}{c}{ Superficie } \\
\cline { 2 - 11 } Rango de CBI & \multicolumn{3}{c}{ Nilahue-Barahona } & \multicolumn{7}{c}{ Las Máquinas } \\
\cline { 2 - 11 } & \multicolumn{2}{c}{ Bosque nativo } & \multicolumn{1}{c}{ Plantación } & \multicolumn{1}{c}{ Bosque nativo } & Plantación & Bosque mixto \\
\cline { 2 - 11 } & $\mathrm{km}^{2}$ & $\%$ & $\mathrm{~km}^{2}$ & $\%$ & $\mathrm{~km}^{2}$ & $\%$ & $\mathrm{~km}^{2}$ & $\%$ & $\mathrm{~km}^{2}$ & $\%$ \\
\hline Alto $(2<)$ & 28,58 & 13,38 & 53,7 & 30,84 & 51,51 & 17,75 & 457,8 & 32,77 & 17,66 & 21,12 \\
Moderado (1-2) & 125,79 & 58,9 & 84,08 & 48,29 & 130,98 & 45,13 & 510 & 36,51 & 42,12 & 50,39 \\
Bajo $(<1)$ & 59,18 & 27,72 & 36,35 & 20,87 & 107,74 & 37,12 & 429,16 & 30,72 & 23,82 & 28,49 \\
\hline Total & 213,55 & 100 & 174,13 & 100 & 290,23 & 100 & $1.396,96$ & 100 & 83,6 & 100 \\
\hline
\end{tabular}



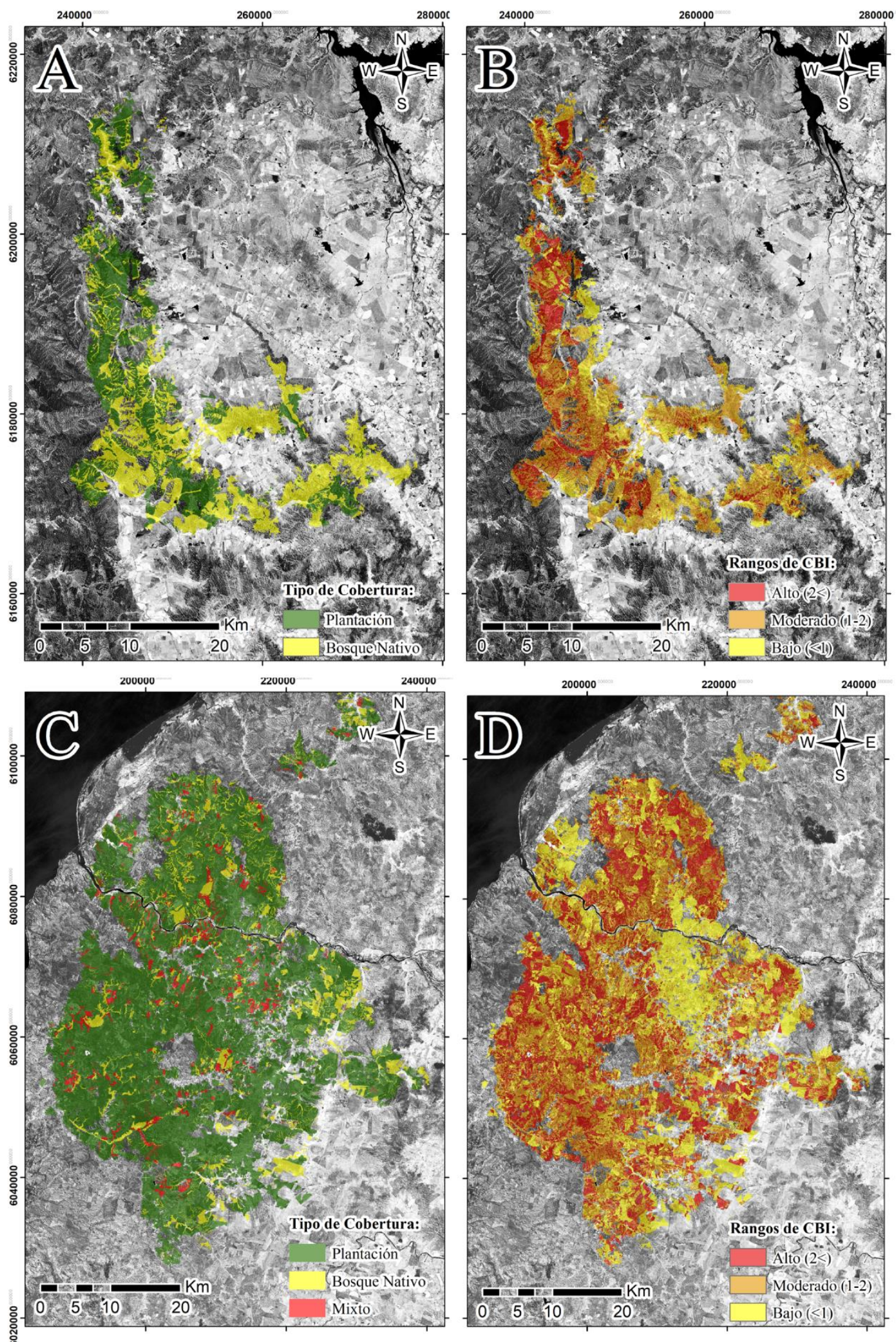

Figura 3. Mapa de tipos de bosque de las áreas Nilahue-Barahona (A)* y Las Máquinas (C)*. Mapa de CBI predicho de acuerdo a tres rangos de severidad del incendio de las áreas Nilahue-Barahona (B) y Las Máquinas (D). *Adaptado de IDE (2018).

Forest types map of Nilahue-Barahona (A)* and Las Máquinas (C)* study areas. Predicted CBI map according to three burn severity ranges of Nilahue-Barahona (B) and Las Máquinas (D) study areas. *Adapted from IDE (2018). 
al. 2018). Por otro lado, las especies nativas de tipo esclerófila tienden a exhibir una relativamente rápida recuperación post-incendio debido a una morfología y fisiología adaptada a condiciones de estrés hídrico y térmico (Gómez y Hahn 2017). Además, el crecimiento post-incendio de las formaciones esclerófilas no solo recae en la diseminación y germinación de semillas, como ocurre en especies como el Pino radiata, sino también en su capacidad de rebrote (Reyes y Casal 2001). Este crecimiento es más rápido en las coberturas herbáceas y arbustivas que conforman el sotobosque, un estrato a menudo inexistente en monocultivos como los aquí estudiados (Key y Benson 2006). Por último, durante la campaña de terreno fue observado un extensivo clareo post-incendio en muchas plantaciones forestales incendiadas, dejando expuestos suelos desnudos bajo preparación. Esta situación contribuye a que los índices espectrales de fechas post-incendio arrojen bajos valores y, por lo tanto, amplias diferencias al ser restados a su correspondiente fecha ex-ante, pudiendo ser interpretadas por el modelo como coberturas severamente incendiadas.

Las diferencias de índices tienen un rendimiento similar entre tipos, lo que indica que aun cuando son sensibles a diferentes parámetros físico-químicos de la vegetación (e.g., vigor y turgencia), existe una alta autocorrelación entre estos, como lo han señalado estudios previos (Jones y Vaughan 2010, Chen et al. 2011, Peña y Ulloa 2017). Aquellas diferencias basadas en el NDWI y NBR presentan altas correlaciones y el mayor poder predictivo para las mediciones hechas en el dosel arbóreo superior, en especial, cuando integran la segunda y tercera fecha postincendio, más coincidentes con la fecha en que fueron colectados los datos de terreno. Ambos índices espectrales se construyen con bandas pertenecientes a la región espectral del infrarrojo de onda corta, que ha mostrado ser de utilidad en varios estudios forestales (Frolking et al. 2009, Banskota et al. 2014), en particular, aquellos orientados a valorar el daño ocasionado por los incendios forestales (Chuvieco y Kasischke 2007, Chen et al. 2011, Tanase et al. 2011, Veraverbeke et al. 2011). La utilidad de estas bandas se debe a su relativamente menor sensibilidad al ruido producido por esparcimiento atmosférico y a su alta sensibilidad para detectar suelos escasamente vegetados o desnudos (Banskota et al. 2014). Por su parte, las correlaciones consistentemente más bajas arrojadas por las diferencias de índices calculadas inmediatamente después de la extinción del fuego se explican por su desfase temporal en relación con la colección de datos de terreno, efectuada dos años después de ocurridos los incendios, cuando los bosques ya estaban recuperados en alguna medida.

Este desfase entre datos teledetectados y de terreno también contribuye a explicar las generalizadas bajas correlaciones arrojadas por el CBI (cuadro 4). Como cabría esperar, el sotobosque se encontraba bastante recuperado al momento de colección de los datos de terreno, reduciendo con ello el valor promedio de daño que entrega el CBI. Por su parte, el dosel arbóreo superior exhibió follaje más dañado en ese momento, especialmente, en sus árboles más robustos y altos, de recuperación más lenta de biomasa. De esta manera, dado que planimetría de las imágenes representa de mejor manera los estratos vegetales superiores, las mediciones efectuadas en terreno sobre el dosel arbóreo superior se correlacionan más fuertemente con los datos derivados de sus píxeles correspondientes (De Santis y Chuvieco 2009). Estos hallazgos son concordantes con los discutidos por French et al. (2008), quienes luego de una exhaustiva revisión bibliográfica referida a la relación del CBI con el NBR y su diferencia en regiones boreales de Norte América, encuentran importantes discrepancias entre los resultados de estudios sujetos a similares condiciones de sitio y metodológicas. Dentro de las causas que explicarían tales discrepancias, los autores destacan la relevancia diferenciada que pueden tener los estratos vegetales y las variables medidas en estos mediante el CBI, en atención a las particularidades ecológicas del ecosistema forestal incendiado y su interacción con la naturaleza y comportamiento del fuego mismo. Lo anterior, podría introducir subestimaciones o sobrestimaciones del CBI; creado como un método estándar de medición de un conjunto acotado de variables de daño biofísico que se promedian a lo largo de los estratos vegetales de la parcela forestal medida, pudiendo contribuir a oscurecer su relación con un índice espectral o su diferencia bitemporal. Asimismo, los autores consignan que la naturaleza planimétrica y rasterizada de los datos remotos tendría por efecto visibilizar más acentuadamente la señal espectral proveniente del dosel arbóreo superior, lo cual sumado a una colección relativamente tardía de datos de $\mathrm{CBI}$, podría sumarse a explicar esas discrepancias. A partir de lo anterior, concluyen que en casos como los por ellos abordados resulta recomendable adaptar la medición del CBI.

En concordancia con lo anterior, el presente estudio revela que el modelo de severidad de incendio más robusto resulta efectivamente de modificar la medición original de $\mathrm{CBI}$, considerando aisladamente las mediciones de terreno efectuadas en el dosel arbóreo superior como variable regresada. Esto justifica la necesidad de diseñar una aproximación empírica de modelado, capaz de adaptarse a las particularidades de cada caso de estudio, así como a la naturaleza de los datos empleados.

Otro aspecto que podría mermar el rendimiento de un modelo como el aquí expuesto, dice relación con la presencia generalizada de altos valores de CBI $(>2,5)$, que pudieran incurrir en la saturación de los índices, haciendo más adecuado el uso de regresiones no lineales (Lentile et al. 2006, French et al. 2008, Tanase et al. 2011). Sin embargo, debido a que la campaña de terreno fue efectuada luego de dos años de ocurridos los incendios, las mediciones hechas en los estratos vegetales, excepcionalmente, se acercaron al límite superior de la escala de daño del CBI (datos no mostrados). Más aún, el rendimiento del modelo aquí presentado $\left(R^{2}\right.$ ajustado $\left.=0,77\right)$ concuerda con aquellos arrojados tanto por regresiones lineales y no lineales 
empleadas en estudios similares (Chen et al. 2011, Veraverbeke et al. 2011, Arellano et al. 2017). No obstante, el diseño de un modelo a partir de una regresión paso a paso posibilita controlar de mejor modo (que en una regresión no selectiva lineal o no lineal) su potencial sobreajuste y multicolinialidad producto de la inclusión de regresores irrelevantes (James et al. 2013). Esto permite la inclusión de un mayor número de potenciales predictores, a pesar de mantener fijo $n$, permitiendo un mayor aprovechamiento de los diferentes proxies de severidad de un incendio forestal que puede proveer un conjunto temporal de imágenes ópticas.

Por último, la presencia de inconsistencias en las señales espectrales que recogen los diferentes índices, también podría impactar negativamente en el rendimiento del modelo, sin embargo, estas han mostrado tener un efecto apreciable a altas latitudes, donde el característico bajo ángulo de elevación solar introduce distorsiones en el comportamiento de la radiancia o flujo radiante ascendente, que son insuficientemente controladas por los modelos de corrección atmosférica. En estos casos es sugerible aplicar correcciones de iluminación topográfica sobre las imágenes, incluso, si se pretende usar índices espectrales como los de este trabajo, que por su formulación normalizada minimizan la variabilidad lumínica resultante de la interacción entre el relieve y la posición solar (French et al. 2008, Jones y Vaughan 2010).

Próximos estudios podrían profundizar y mejorar la metodología aquí expuesta mediante la exploración de otros modelos de regresión, como aquellos de aprendizaje automático basados en la regularización de la recta de ajuste, capaces de incorporar un número mucho mayor de predictores en comparación con aquellos tradicionalmente basados en ajuste por mínimos cuadrados, y que en aplicaciones de ecología vegetal muestran promisorios resultados (Zandler et al. 2015). Además, es recomendable explorar el impacto que tendría una posible autocorrelación espacial de los regresores en la predictibilidad del modelo, para lo cual la partición efectuada sobe los datos de la variable regresora en el procedimiento de validación cruzada, debería realizarse en atención a su vecindad espacial. En un estudio comparativo del rendimiento de seis conocidos modelos de aprendizaje automático aplicados a un caso de estudio de enfermedades en bosques, Schratz et al. (2019) demuestran que, en presencia de autocorrelación espacial del predictor, la validación cruzada arroja consistentemente precisiones sobreoptimistas, haciéndose necesaria una partición "espacial" de sus datos.

El modelo aquí planteado podría incorporar datos de CBI pertenecientes a otras áreas forestadas recientemente incendiadas en Chile central y sujetas a distintas condiciones de sitio. Esto ampliaría la aplicabilidad del modelo a diversas condiciones geográficas y ecológicas de Chile central, robusteciendo su predictibilidad. El acometido de estas tareas cobra especial significado en el contexto climático de Chile central, donde los incendios forestales han mostrado un aumento en frecuencia e intensidad producto de la acentuación de la variabilidad climática a la que responde su disponibilidad de biomasa y combustible (disminución de precipitaciones invernales y aumento de sequías estivales durante años previos al incendio, en respuesta a El Niño-Oscilación del Sur y la fase positiva de la Oscilación Antártica, respectivamente) (Peña-Fernández y Valenzuela-Palma 2004, González et al. 2011, Urrutia et al. 2018).

\section{CONCLUSIONES}

Este estudio modela la severidad de dos de los mayores incendios forestales que se tenga registro en Chile: Nilahue-Barahona y Las Máquinas, ocurridos durante el verano de 2016-17, a partir de una regresión paso a paso basada en diferencias aritméticas entre fechas ex-ante y ex-post de índices espectrales sensibles a los estados de vigor, turgencia y calcinación vegetal. El buen rendimiento del modelo construido $\left(R^{2}\right.$ ajustado $=0,77 ;$ RMSE $=$ 0,35 ) es semejable a aquellos obtenidos mediante regresiones lineales y no lineales en áreas forestadas conformadas por especies y condiciones de sitio distintas, facultando transferirlo a una cartografía capaz de proveer una visión espacial sinóptica y detallada de los ecosistemas forestales perturbados.

El mejor modelo se construye con cuatro de las nueve diferencias de índices empleadas como regresoras, todas están basadas en el NDWI y el NBR que, al emplear bandas del infrarrojo de onda corta en su formulación, destacan por su particular sensibilidad al estrés hídrico vegetal inducido por el fuego. Estas diferencias de índices incorporan fechas ex-post preferentemente más recientes $\mathrm{y}$, por lo tanto, más coincidentes con el daño observado en las parcelas forestales durante la campaña de terreno.

Tal como advierte la literatura científica, el adecuado modelado de la severidad de un incendio puede requerir adaptar la metodología de CBI a las particularidades ecológicas del área de interés, lo que en este estudio implica el empleo de los datos medidos en el dosel superior de las parcelas forestales muestreadas; más apreciablemente dañado en las imágenes que el sotobosque (en gran medida recuperado al momento de colección de los datos de terreno) y mejor representado desde la planimetría de las imágenes.

La replicación de esta metodología sobre otras áreas forestadas afectadas por incendios en Chile central, incorporando mejoras como la implementación de validaciones cruzadas espaciales, contribuiría a robustecer el modelo aquí expuesto.

\section{AGRADECIMIENTOS}

Este estudio fue financiado por el Fondo de Fomento a la Investigación de la Universidad Alberto Hurtado (DIP2019-11). 


\section{REFERENCIAS}

Arellano S, JA Vega, F Rodríguez y Silva, C Fernández, D VegaNieva, JG Álvarez-González, AD Ruiz-González. 2017. Validación de los índices de teledetección dNBR y RdNBR para determinar la severidad del fuego en el incendio forestal de Oia-O Rosal (Pontevedra) en 2013. Revista de Teledetección 49 Special Issue: 49-61. DOI: https://doi. org/10.4995/raet.2017.7137

Banskota A, N Kayastha, MJ Falkowski, MA Wulder, RE Froese, JC White. 2014. Forest monitoring using Landsat time series data: A review. Canadian Journal of Remote Sensing 40(5): 362-384. DOI: https://doi.org/10.1080/07038992.20 $\underline{14.987376}$

Bastos A, CM Gouveia, CC DaCamara, RM Trigo. 2011. Modelling post-fire vegetation recovery in Portugal. Biogeosciences 8(3): 3593-3607. DOI: https://doi.org/10.5194/bg$\underline{\text { 8-3593-2011 }}$

Bowman DMJS, A Moreira-Muñoz, CA Kolden, RO Chávez, AA Muñoz, F Salinas, A González-Reyes, R Rocco, F de la Barrera, GJ Williamson, N Borchers, LA Cifuentes, JT Abatzoglou, FH Johnston. 2018. Human-environmental drivers and impacts of the globally extreme 2017 Chilean fires. Ambio 48(4): 350-362. DOI: https://doi.org/10.1007/ $\underline{\mathrm{s} 13280-018-1084-1}$

Chen X, JE Vogelmann, M Rollins, D Ohlen, CH Key, L Yang, C Huang, H Shi. 2011. Detecting post-fire burn severity and vegetation recovery using multitemporal remote sensing spectral indices and field-collected composite burn index data in a ponderosa pine forest. International Journal of Remote Sensing 32(23): 7905-7927. DOI: https://doi.org/10.1 $\underline{080 / 01431161.2010 .524678}$

Chuvieco E. 1999. Remote sensing of large wildfires: In the European Mediterranean basin. Berlín, Alemania. Springer. 212 p. DOI: https://doi.org/10.1007/978-3-642-60164-4

Chuvieco E, ES Kasischke. 2007. Remote sensing information for fire management and fire effects assessment. Journal of Geophysical Research 112(1): G01S90. DOI: https://doi. org/10.1029/2006jg000230

Chuvieco E, MP Martín, A Palacios. 2002. Assessment of different spectral indices in the red-near-infrared spectral domain for burned land discrimination. International Journal of Remote Sensing 23(23): 5103-5110. DOI: https://doi. org $/ 10.1080 / 01431160210153129$

CONAF (Corporación Nacional Forestal, CL). 2017. Análisis de la afectación y severidad de los incendios forestales ocurridos en enero y febrero de 2017 sobre los usos de suelo y los ecosistemas naturales presente entre las regiones de Coquimbo y Los Ríos de Chile. Santiago, Chile. CONAF. 56 p. https://www.conaf.cl/tormenta de fuego-2017/INFORME-AFECTACION-Y SEVERIDAD-DE-INCENDIOS-FORESTALES-VERANO-2017-SOBRE-ECOSISTEMAS-VEGETACIONALES-CONAF.pdf

De Santis A, E Chuvieco. 2009. GeoCBI: A modified version of the Composite Burn Index for the initial assessment of the short-term burn severity from remotely sensed data. Remote Sensing of Environment 113(3): 554-562. DOI: https:// doi.org/10.1016/j.rse.2008.10.011

Díaz-Delgado R. 2000. Caracterización por medio teledetección del régimen de incendios forestales en Cataluña (período 1975 - 98) y su influencia en los procesos de regeneración.
Tesis Doctoral Ciencias Biológicas. Barcelona, España. Facultad de Ciencias, Universitat Autònoma Barcelona. 276 p.

Díaz-Hormazábal I, ME González. 2016. Análisis espacio-temporal de incendios forestales en la región del Maule, Chile. Bosque 37(1): 147-158. DOI: https://doi.org/10.4067/ s0717-92002016000100014

Escuín S, R Navarro, P Fernández. 2008. Fire severity assessment by using NBR (normalized burn ratio) and NDVI (normalized difference vegetation index) derived from Landsat TM/ETM images. International Journal of Remote Sensing 29(4): 1053-1073. DOI: https://doi. org/10.1080/01431160701281072

ESA (European Space Agency, FR) (2020). Copernicus Open Access Hub. European Space Agency. Consultado 20 ene. 2021. Disponible en https://scihub.copernicus.eu/

French NHF, ES Kasischke, RJ Halle, KA Murphy, DL Verbyla, EE Hoy, JL Allen. 2008. Using Landsat data to assess fire and burn severity in the North American boreal forest region: an overview and summary of results. International Journal of Wildland Fire 17(4): 443-462. DOI: https://doi. org/10.1071/wf08007

Frolking S, MW Palace, DB Clark, JQ Chambers, HH Shugart, GC Hurtt. 2009. Forest disturbance and recovery: A general review in the context of spaceborne remote sensing of impacts on aboveground biomass and canopy structure. Journal of Geophysical Research 114(3): G00E02. DOI: https:// doi.org/10.1029/2008jg000911

Gao B. 1996. NDWI-A normalized difference water index for remote sensing of vegetation liquid water from space. $R e$ mote Sensing of Environment 58(3): 257-266. DOI: https:// doi.org/10.1016/s0034-4257(96)00067-3

Gitas I, G Mitri, S Veraverbeke, A Polychronaki. 2012. Advances in remote sensing of post-fire vegetation recovery monitoring - A review. In Fatoyinbo $\mathrm{T}$ ed. Remote sensing of biomass: Principles and applications. London, U.K. Intech Open Limited. p. 143-176. DOI: https://doi.org/10.5772/20571

Gómez P, S Hahn. 2017. Regeneración posincendio de plantas leñosas en plantaciones de Pinus radiata D. Don, zona costera, Región del Maule, Chile central. Gayana Botánica 74(2): 302-3016. DOI: http://dx.doi.org/10.4067/S071766432017000200302

González ME, A Lara, R Urrutia, J Bosnich. 2011. Cambio climático y su impacto potencial en la ocurrencia de incendios forestales en la zona centro-sur de Chile $\left(33^{\circ}-42^{\circ} \mathrm{S}\right)$. Bosque 32(3): 215-219. DOI: https://doi.org/10.4067/s071792002011000300002

Hunt ER, BN Rock, PS Nobel. 1987. Measurement of leaf relative water content by infrared reflectance. Remote Sensing of Environment 22(3): 429-435. DOI: https://doi. org/10.1016/0034-4257(87)90094-0

IDE (Infraestructura de Datos Geoespaciales, CL). 2018. Catastros de uso de suelo y vegetación. Consultado 11 may. 2020. Disponible en http://www.ide.cl/index.php/flora-y-fauna/ item/1513-catastros-de-uso-de-suelo-y-vegetacion

James G, D Witten, T Hastie, R Tibshirani. 2013. An introduction to statistical learning: With applications in R. New York, USA. Springer Texts in Statistics. 426 p. DOI: https://doi. org/10.1007/978-1-4614-7138-7_1

Jones G, R Vaughan. 2010. Remote sensing of vegetation: Principles, techniques, and applications. New York, USA. Oxford University Press. 353 p. 
Kafka V, G Sylvie, B Yves. 2001. Fire impacts and crowning in the boreal forest: study of a large wildfire in western Quebec. International Journal of Wildland Fire 10(2), 119-127. DOI: https://doi.org/10.1071/WF01012

Keeley JE. 2009. Fire intensity, fire severity and burn severity: a brief review and suggested usage. International Journal of Wildland Fire 18(1): 116-126. DOI: https://doi. org/10.1071/wf07049

Key CH, NC Benson. 2006. Landscape Assessment (LA): Sampling and analysis methods. In Lutes DC, RE Keane, JF Caratti, CH Key, NC Benson, S Sutherland, LJ Gangi eds. FIREMON: Fire effects monitoring and inventory system. Rocky Mountain, USA. USDA Forest Service General Technical Report RMRS-GTR-164-CD. p 219-273. DOI: https://doi.org/10.2737/rmrs-gtr-164

Lentile LB, ZA Holden, AMS Smith, MJ Falkowski, AT Hudak, P Morgan, SA Lewis, PE Gessler, NC Benson. 2006. Remote sensing techniques to assess active fire characteristics and post-fire effects. International Journal of Wildland Fire 15(3), 319-345. DOI: https://doi.org/10.1071/ wf05097

Oliveira S, F Félix, A Nunes, L Lourenço, G Laneve, A SebastiánLópez. 2018. Mapping wildfire vulnerability in Mediterranean Europe. Testing a stepwise approach for operational purposes. Journal of Environmental Management 206, 158169. DOI: https://doi.org/10.1016/j.jenvman.2017.10.003

Peña-Fernández E, L Valenzuela-Palma. 2004. Incremento de los incendios forestales en bosques naturales y plantaciones forestales en Chile. Memorias del Segundo Simposio Internacional sobre Políticas, Planificación y Economía de los Programas de Protección contra Incendios Forestales: Una Visión Global, 19-22 Abril, 2004, Córdoba, España.

Peña MA, A Brenning, A Sagredo. 2012. Constructing satellitederived hyperspectral indices sensitive to canopy structure variables of a Cordilleran Cypress (Austrocedrus chilensis) forest. ISPRS Journal of Photogrammetry \& Remote Sensing 74: 1-10. DOI: https://doi.org/10.1016/j.isprsjprs.2012.06.010

Peña MA, R Liao, A Brenning. 2017. Using spectrotemporal indices to improve the fruit-tree crop classification accuracy. ISPRS Journal of Photogrammetry \& Remote Sensing 128:158-169. DOI: https://doi.org/10.1016/j.isprsjprs.2017.03.019

Peña MA, G Martínez. 2019. Evaluación del impacto del incendio Nilahue-Barahona mediante índices espectrales ex-ante y expost. Revista Geográfica Terra Australis 55(1): 1-11. DOI: https://doi.org/10.23854/07199562.2019551.penal

Peña MA, J Ulloa. 2017. Mapeo de la recuperación de la vegetación quemada mediante la clasificación de índices espectrales pre- y post-incendio. Revista de Teledetección 50: 37-48. DOI: https://doi.org/10.4995/raet.2017.7931

Pérez Mato J. 2017. Autonomous wildfire geolocation system based on thermographic and synthetic vision techniques. Tesis Doctoral en Cibernética y Telecomunicación. Gran Canaria, España. Facultad de Ingeniería en Telecomunicación y Electrónica, Universidad de Las Palmas de Gran Canaria. 160 p.
Plana E, M Font, M Serra, M Borràs, O Vilalta. 2016. El fuego y los incendios forestales en el Mediterráneo; la historia de una relación entre bosques y sociedad. Cinco mitos y realidades para saber más. Cataluña, España. Ediciones CTFC. 36 p.

Reyes, O, M Casal. 2001. The influence of seed age on germinative response to the effects of fire in Pinus pinaster, Pinus radiata and Eucalyptus globulus. Annals of Forest Science 58(4): 439-447. DOI: https://doi.org/10.1051/forest: 2001137

Rouse JW, RH Haas, JA Schell, DW Deering. 1973. Monitoring vegetation systems in the Great Plains with ERTS. In Stanley CF, PM Enrico, AB Margaret eds. Third Earth resources technology satellite Symposium. Washington, USA. NASA SP-351 I. p. $309-317$.

Ryu JH, KS Han, S Hong, NW Park, YW Lee, J Cho. 2018. Satellite-based evaluation of the post-fire recovery process from the worst forest fire case in South Korea. Remote Sensing 10(6): 918. DOI: https://doi.org/10.3390/rs10060918

Schratz P, J Muenchow, E Iturritxa, J Richter, A Brenning. 2019. Hyperparameter tuning and performance assessment of statistical and machine-learning algorithms using spatial data. Ecological Modelling 406: 109-120. DOI: https://doi. org/10.1016/j.ecolmodel.2019.06.002

Sever L, J Leach, L Bren. 2012. Remote sensing of post-fire vegetation recovery; a study using Landsat 5 TM imagery and NDVI in North-East Victoria. Journal of Spatial Science 57(2): 175-191. DOI: https://doi.org/10.1080/14498596.20 $\underline{12.733618}$

Tanase M, J de la Riva, F Pérez-Cabello. 2011. Estimating burn severity at the regional level using optically based indices. Canadian Journal of Forest Research 41(4): 863-872. DOI: https://doi.org/10.1139/x11-011

Urrutia-Jalabert R., M González, Á González-Reyes, A Lara, R Garreaud. 2018. Climate variability and forest fires in central and south-central Chile. Ecosphere 9(4): 1-17. DOI: https://doi.org/10.1002/ecs2.2171

Veraverbeke S, S Lhermitte, WW Verstraeten, R Goossens. 2011. Evaluation of pre/post-fire differenced spectral indices for assessing burn severity in a Mediterranean environment with Landsat Thematic Mapper. International Journal of Remote Sensing, 32(12): 3521-3537. DOI: https://doi. org/10.1080/01431161003752430

Westerling AL, HG Hidalgo, DR Cayan, TW Swetnam. 2006. Warming and earlier spring increase western U.S. forest wildfire activity. Science 313(5789): 940-943. DOI: https://doi.org/10.1126/science.1128834

Zandler H, A Brenning, C Samimi. 2015. Quantifying dwarf shrub biomass in an arid environment: comparing empirical methods in a high dimensional setting, Remote Sensing of Environment, 158: 140-155. DOI: https://doi.org/10.1016/j. rse.2014.11.007

Zhu Z, C Key, D Ohlen, N Benson. 2006. Evaluate sensitivities of burn severity mapping algorithms for different ecosystems and fire histories in the United States. Sioux Falls, USA. US Department.of Interior, Final Report to the Joint Fire Science Program: Project JFSP 01-1-4·12. States. 35 p. 
